\title{
Hepatic steatosis in obese children and adolescents
}

\author{
Maria Amélia S. M. Duarte, ${ }^{1}$ Giselia Alves Pontes da Silva ${ }^{2}$
}

\begin{abstract}
Objective: To assess the frequency of hepatic steatosis and metabolic syndrome among obese children and adolescents.

Method: A descriptive case series was conducted with 77 patients, aged 2 to 13 years and 11 months, who were followed up from February to July 2007. Obesity was defined as body mass index $\geq P_{95}$ adjusted for age and sex. Liver ultrasound was performed to diagnose hepatic steatosis. Metabolic syndrome was defined according to the modified criteria suggested by Cook et al.

Results: Hepatic steatosis was diagnosed in 33/77 patients (42.9\%), 25/33 (75.8\%) with mild steatosis and $8 / 33(24.2 \%)$ with moderate steatosis. Those aged less than 10 years showed only mild steatosis, and the moderate degree of the disease was restricted to adolescents. Aminotransferase alterations were found in $9.1 \%(3 / 33)$ of patients with hepatic steatosis and in $4.9 \%(2 / 41)$ of those without the disorder. Mean waist circumference was $84.74 \pm 2.84 \mathrm{~cm}$ for patients with hepatic steatosis and $78.24 \pm 1.60 \mathrm{~cm}$ for those without the disease $(p=0.04)$. Metabolic syndrome was diagnosed in $27.3 \%$ (21/77) of obese patients, $47.6 \%(10 / 21)$ of them having steatosis, $60 \%$ had mild steatosis and $40 \%$ had a moderate degree of the disorder.

Conclusions: The frequency of hepatic steatosis and metabolic syndrome was high. The association of larger waist circumference with hepatic steatosis highlights the importance of taking this parameter into consideration when investigating obese patients.

J Pediatr (Rio J). 2011;87(2):150-156: Obesity, hepatic steatosis, metabolic syndrome, children, adolescent.
\end{abstract}

\section{Introduction}

Prevalence of childhood obesity has been growing fast in recent decades worldwide, reaching the status of an epidemic. ${ }^{1}$ Hepatic steatosis, which is the initial stage of nonalcoholic fatty liver disease (NAFLD), is one of the most important effects caused by obesity on children and adolescents' health. $^{2}$

NAFLD is an emerging clinical problem among young and adult obese patients. ${ }^{3,4}$ It is a syndrome of multifactorial etiology in which obesity is the most common associated factor. ${ }^{5}$ In addition, NAFLD has a broad spectrum of hepatic histological changes. Initially, there is liver fat infiltration (steatosis), which may progress to disease activity and necrosis, with or without perisinusoidal fibrosis (steatohepatitis), advanced fibrosis, and cirrhosis.6,7 Early diagnosis of hepatic steatosis is important because $28 \%$ of patients that progress to steatohepatitis may have cirrhosis and hepatic carcinoma, being at a high risk of needing a liver transplant. ${ }^{7}$

1. Mestre, Saúde da Criança e do Adolescente, Universidade Federal de Pernambuco (UFPE), Recife, PE. Serviço de Endocrinologia Pediátrica, Hospital Barão de Lucena, Recife, PE, Brazil.

2. Doutora, Pediatria, Escola Paulista de Medicina (EPM), Universidade Federal de São Paulo (UNIFESP), São Paulo, SP, Brazil. Departamento Materno-Infantil, Centro de Ciências da Saúde, UFPE, Recife, PE, Brazil.

No conflicts of interest declared concerning the publication of this article.

Financial support: Fundação de Amparo à Ciência e Tecnologia do estado de Pernambuco (FACEPE).

Suggested citation: Duarte MA, Silva GAP. Hepatic steatosis in obese children and adolescents. J Pediatr (Rio J). 2011;87(2):150-156.

Manuscript submitted Sep 1, 2010, accepted for publication Nov 3, 2010.

doi:10.2223/JPED.2065 
NAFLD should be suspected when there is hepatomegaly on physical examination, increased echogenicity on ultrasound, suggesting accumulation of lipids in the liver, or a slight (two to three times higher than the reference value) and persistent (two or more occasions) increase in aminotransferase in individuals without any apparent cause (exclusion of other liver diseases). ${ }^{8}$

Nevertheless, these diagnostic methods are usually insufficient to establish the diagnosis. Based on findings suggestive of steatosis on ultrasound, it is not possible to differentiate between simple steatosis and steatohepatitis, and aminotransferase levels may be normal. ${ }^{2}$

The histological examination of a liver fragment is considered to be the gold standard method for assessment, but it is not done often in children and adolescents because it is an invasive technique. ${ }^{6}$ Therefore, in this population, clinical, biochemical and ultrasound diagnoses of hepatic steatosis remain as the methods of choice. ${ }^{6}$

The first report of children with NAFLD was first published in $1983^{\circ}$ in obese children, but NAFLD has become a relatively common cause of liver disease as a result of the increase in the number of obese people. Prevalence of NAFLD in obese children and adolescents ranges between 22.5 and $77 \%$ when diagnosed by means of ultrasound. ${ }^{10,11}$

The objective of the present study is to verify the frequency of hepatic steatosis and metabolic syndrome among obese children and adolescents.

\section{Methods}

We conducted a descriptive case series at the pediatric endocrinology center of Hospital Barão de Lucena, in Recife (state of Pernambuco), Brazil. All children and adolescents treated between February and July 2007 and who had body mass index (BMI) $\geq \mathrm{P}_{95}$ for age and sex according to the charts of the Center for Disease Control and Prevention were included in the study. ${ }^{12}$ We analyzed the clinical and laboratory characteristics of 77 patients of both sexes who were younger than 14 years of age, had a diagnosis of obesity, and whose guardians signed the written consent form. We excluded patients on therapy with corticosteroids or estrogen, as well as those who had a diagnosis of metabolic disease causing hepatic steatosis or history of alcohol abuse.

Clinical variables included: waist circumference, blood pressure, and presence of acanthosis nigricans. Laboratory variables were: serum levels of alanine and aspartate aminotransferase, total cholesterol and cholesterol fractions, fasting triglycerides and glucose, in addition to ultrasound diagnosis of hepatic steatosis. Metabolic syndrome was diagnosed based on the modified criteria suggested by Cook et al., ${ }^{13}$ considering: triglycerides $\geq 110 \mathrm{mg} / \mathrm{dL}$, glycemia $>100 \mathrm{mg} / \mathrm{dL}, \mathrm{HDL} \leq 40 \mathrm{mg} / \mathrm{dL}$, systolic or diastolic blood pressure $\geq P_{90}$, and waist circumference $\geq P_{90}$ for age and sex.

A single measurement of weight was carried out using a platform-type calibrated scale with the individual standing erect on the center of the scale, barefoot, heels together, straight back, arms stretched along the body, and wearing light clothing. Height was measured using a stadiometer. BMI was calculated based on the Quetelet index (BMI = weight/height ${ }^{2}$ ).

Waist circumference was measured using a nonextensible tape measure that circled the patient while standing erect, using the midpoint between the last rib and the iliac crest as reference, measuring the circumference in centimeters during expiration. Waist circumference was measured according to the guidelines for African-American children and adolescents of the International Diabetes Federation. ${ }^{14}$

Blood pressure was measured after the patient received information on the procedure to ensure reduced stress and anxiety. With the patient sitting at rest for 3 to 5 minutes, the arm supported at the same level as the precordium, we measured blood pressure in the right arm using a mercury sphygmomanometer equipped with a cuff according to the child's size. The mean of both blood pressure measurements was used as reference. The classification of systolic or diastolic hypertension was according to the parameters of the $\mathrm{V}$ Brazilian Guidelines on Hypertension. ${ }^{15}$

The diagnosis of acanthosis nigricans was based on the presence of hyperchromic spots on skin folds and neck. ${ }^{16}$

Criteria for interpretation of laboratory measurements were: Kwiterovich ${ }^{17}$ for serum lipid profile; International Diabetes Federation ${ }^{14}$ for fasting glycemia; and Seist et al. ${ }^{18}$ for aminotransferase determined by enzyme kinetics.

Hepatic steatosis was identified on ultrasound using display C, Philips ${ }^{\circledR}, 2-5 \mathrm{MHz}$ convex transducer. The procedure was carried out by a physician with specialization in diagnostic imaging. Liver fat infiltration was stratified into scores ranging from one to nine, as shown in Table 1. The categorization used was: normal liver (zero points), mild diffuse steatosis (1-3 points), moderate steatosis (4-6 points), and severe steatosis (7-9 points).

After the guardians signed the written consent form, data collection of each patient was initiated by means of interview with their guardians. Next, the participants underwent physical examination, laboratory tests, and ultrasound. All patients received clinical follow-up during the study and continued to be monitored at the outpatient clinic later.

Data analysis was performed using Epi-Info version 2000. To analyze differences between the groups with or without steatosis, mean, standard deviation, and distribution of absolute and relative frequencies were used. For contingency analysis and mean difference, we used 
Table 1 - Ultrasonographic parameters for assessment of hepatic steatosis

\begin{tabular}{|c|c|}
\hline Ultrasonographic aspects/score & Definition of degrees of fat infiltration \\
\hline \multicolumn{2}{|l|}{ Hepatic echotexture } \\
\hline 0 & $\begin{array}{l}\text { Normal (normal liver parenchyma echo and no difference in the contrast } \\
\text { between liver and kidney parenchymas) }\end{array}$ \\
\hline 1 & Mild (slight increase in hepatic echotexture) \\
\hline 2 & Moderate (hepatic echotexture between degrees 1 and 3 ) \\
\hline 3 & $\begin{array}{l}\text { Severe (marked discrepancy in the echogenicity of liver parenchyma } \\
\text { in relation to the renal parenchyma) }\end{array}$ \\
\hline \multicolumn{2}{|c|}{ Penetration of the echo and visibility of the diaphragm } \\
\hline 0 & $\begin{array}{l}\text { Normal (the hepatic structure is clearly distinguishable from the surface } \\
\text { of the diaphragm, whose borders can be easily identified) }\end{array}$ \\
\hline 1 & Mild (slight echo attenuation in the liver) \\
\hline 2 & Moderate (intermediate between degrees 1 and 3 ) \\
\hline 3 & $\begin{array}{l}\text { Severe (pronounced echo attenuation in the liver, diaphragm } \\
\text { cannot be identified) }\end{array}$ \\
\hline \multicolumn{2}{|l|}{ Distinctness of the hepatic vasculature } \\
\hline 0 & Normal (walls and lumen of the vessels are clearly identified) \\
\hline 1 & Mild (slight reduction in the definition of the walls of portal venules) \\
\hline 2 & Moderate (intermediate between degrees 1 and 3 ) \\
\hline 3 & $\begin{array}{l}\text { Severe (only the walls of the main portal vein may be visualized, } \\
\text { while the walls of the smaller portal veins are not visible) }\end{array}$ \\
\hline
\end{tabular}

Source: adapted from Tominaga et al. ${ }^{10}$

the chi-square test and Student's $t$ test, respectively, at a significance level of 0.05 .

This study was approved by the Research Ethics Committee of the Center of Health Sciences (CEP/CCS) of Universidade Federal de Pernambuco (UFPE) under protocol CEP/CCS/UFPE 247/06.

\section{Results}

We investigated 77 patients, 26 (33.8\%) of them were females and $51(66.2 \%)$ males, aged between 2 years and 13 years and 11 months, living in Recife and the metropolitan area, state of Pernambuco, Brazil.

Hepatic steatosis was diagnosed in $42.9 \%$ (33/77) of the sample. We found a higher frequency of mild steatosis (25/33), which was more prevalent among school-age children $(18 / 24,75 \%)$, while the moderate degree of the disease was restricted to adolescents.

Among those patients with steatosis, most were male $(24 / 33,72.7 \%)$ and children under 10 years of age $(18 / 33$, $54.5 \%)$; acanthosis nigricans was identified in $81.8 \%$ of patients (27/33). Blood pressure was abnormal in $45.2 \%$ $(14 / 33)$ of the patients with hepatic steatosis and in $38.6 \%$ $(17 / 44)$ of those without steatosis. Among the cases with steatosis, stage 2 hypertension was the most frequent variable $(7 / 33,22.6 \%)$ (Table 2$)$.
Mean waist circumference was significantly larger among obese patients with hepatic steatosis $(84.74 \pm 2.84 \mathrm{~cm})$ than among patients without steatosis $(78.24 \pm 1.60 \mathrm{~cm})$ $(p=0.04)$.

There were aminotransferase alterations in 9.1\% (3/33) of the patients with steatosis and in $4.9 \%(2 / 44)$ of those without the disease. All patients had normal glycemia. The evaluation of the lipid profile revealed a higher frequency of high total cholesterol (39.5\%). We found a prevalence of hypercholesterolemia (total cholesterol or LDL) and hypertriglyceridemia associated with steatosis. The reduction in HDL cholesterol was more frequent in those patients without steatosis $(29.3 \%)$ than in patients with steatosis $(9.1 \%)$, and this difference was significant $(p=0.03)$ (Table 3).

Metabolic syndrome was diagnosed in $27.3 \%(21 / 77)$ of patients, of whom $47.6 \%(10 / 21)$ had steatosis, $60 \%$ of them with mild degree and $40 \%$ showing a moderate degree.

In the group with steatosis, five out of the 18 children $(27.8 \%)$ under 10 years old had metabolic syndrome identified by the association of three of the following parameters: increased waist circumference, hypertension, decreased $\mathrm{HDL}$, and hypertriglyceridemia.

The diagnosis of metabolic syndrome was established in 10 out of the 33 (30.3\%) patients with hepatic steatosis 
Table 2 - Distribution of variables in 77 obese children and adolescents according to the presence of hepatic steatosis

\begin{tabular}{|c|c|c|c|c|}
\hline \multirow[b]{2}{*}{ Variables } & \multicolumn{2}{|c|}{ Hepatic steatosis } & \multirow[b]{2}{*}{ Total, n (\%) } & \multirow[b]{2}{*}{$\mathbf{p}$} \\
\hline & Yes, n (\%) & No, n (\%) & & \\
\hline Sex & & & & 0.30 \\
\hline Male & $24(72.7)$ & $27(61.4)$ & $51(66.2)$ & \\
\hline Female & $9(27.3)$ & $17(38.6)$ & $26(33.8)$ & \\
\hline Age (years) & & & & 0.71 \\
\hline$<6$ & $7(21.2)$ & $10(22.7)$ & $17(22.0)$ & \\
\hline 6 to 9 & $11(33.3)$ & $18(40.9)$ & $29(37.7)$ & \\
\hline 10 to 14 & $15(45.5)$ & $16(36.4)$ & $31(40.3)$ & \\
\hline Diagnosis of acanthosis nigricans & & & & 0.08 \\
\hline Yes & $27(81.8)$ & $28(63.6)$ & $55(71.4)$ & \\
\hline No & $6(18.2)$ & $16(36.4)$ & $22(28.6)$ & \\
\hline Blood pressure* & & & & 0.43 \\
\hline Normal & $17(54.8)$ & $27(61.4)$ & $44(58.6)$ & \\
\hline Threshold & $3(9.7)$ & $6(13.6)$ & $9(12.0)$ & \\
\hline Stage 1 & $4(12.9)$ & $7(15.9)$ & $11(14.7)$ & \\
\hline Stage 2 & $7(22.6)$ & $4(9.1)$ & $11(14.7)$ & \\
\hline
\end{tabular}

* We could not measure blood pressure of two cases with hepatic steatosis because the patients refused to cooperate.

Table 3 - Distribution of laboratory variables in 77 children and adolescents according to the presence of hepatic steatosis

\begin{tabular}{|c|c|c|c|c|}
\hline \multirow[b]{2}{*}{ Laboratory variables } & \multicolumn{2}{|c|}{ Hepatic steatosis } & \multirow[b]{2}{*}{ Total, n (\%) } & \multirow[b]{2}{*}{$\mathbf{p}$} \\
\hline & Yes, n (\%) & No, n (\%) & & \\
\hline \multicolumn{5}{|l|}{ Glycemia } \\
\hline Normal & $33(100.0)$ & $44(100.0)$ & $77(100.0)$ & \\
\hline Aspartate aminotransferase* & & & & 0.44 \\
\hline Normal & $32(97.0)$ & $41(100.0)$ & $73(98.6)$ & \\
\hline Abnormal & $1(3.0)$ & - & $1(1.4)$ & \\
\hline Alanine aminotransferase & & & & 0.40 \\
\hline Normal & $30(90.9)$ & $39(95.1)$ & $69(93.2)$ & \\
\hline Abnormal & $3(9.1)$ & $2(4.9)$ & $5(6.8)$ & \\
\hline Cholesterolemia $^{+}$ & & & & 0.73 \\
\hline Normal & $19(57.6)$ & $27(62.7)$ & $46(60.5)$ & \\
\hline Threshold & $10(30.3)$ & $13(30.2)$ & $23(30.3)$ & \\
\hline Increased & $4(12.1)$ & $3(7.1)$ & $7(9.2)$ & \\
\hline HDL cholesterol* & & & & 0.03 \\
\hline Desirable & $30(90.9)$ & $29(70.7)$ & $59(79.7)$ & \\
\hline Decreased & $3(9.1)$ & $12(29.3)$ & $15(20.3)$ & \\
\hline LDL cholesterol $^{+}$ & & & & 0.38 \\
\hline Normal & $22(66.7)$ & $34(79.1)$ & $56(73.7)$ & \\
\hline Threshold & $7(21.2)$ & $7(16.3)$ & $14(18.4)$ & \\
\hline Increased & $4(12.1)$ & $2(4.6)$ & $6(7.9)$ & \\
\hline Triglycerides & & & & 0.26 \\
\hline Normal & $20(60.6)$ & $32(72.7)$ & $52(67.5)$ & \\
\hline Increased & $13(39.4)$ & $12(27.3)$ & $25(32.5)$ & \\
\hline
\end{tabular}

* Percentages calculated based on the number of patients with steatosis (33), patients without steatosis (41), and total number of patients (74).

$\dagger$ We could not assess total cholesterol and LDL in one patient. 
and in 11 out of the $44(25 \%)$ patients without hepatic steatosis; this difference was not significant $(p=0.453)$ (Figure 1).

\section{Discussion}

The frequency of hepatic steatosis we found in the present study $(42.9 \%)$ is high considering the age of the patients involved. Hepatic steatosis with onset in childhood and adolescence should receive special attention because it is manifested by few symptoms and its progression is silent and potentially lethal. In addition to hypertension, diabetes mellitus and dyslipidemia are included among the complications caused by obesity. ${ }^{19}$ The progression of NAFLD can be avoided by means of weight loss, leading to decrease in the degree of liver fat infiltration and reversal of biochemical abnormalities. ${ }^{20}$ Therefore, the early detection of liver fat is important to increase the chances of reversing the damage. 3,11

The frequency of hepatic steatosis in the present case series was lower than that reported by Chan et al., ${ }^{11}$ who diagnosed the disorder using ultrasound in $77 \%$ of 84 patients aged between 7 and 18 years. The authors considered that this high frequency was caused by the fact that the patients were referred by primary care physicians to specialized care due to their high degree of obesity. The differences in terms of ethnicity and age, in addition to the fact that hepatic steatosis is more frequent among Asian people, should be considered when comparing this finding with our data. 3,5

Liver ultrasound is described as a good method for the diagnosis and management of the degree of liver fat infiltration although it does not correlate with the degree of fibrosis. Ultrasound has sensitivity, specificity, positive and negative predictive values of $64,97,96$ and $65 \%$, respectively, for echo pattern of mild hepatic steatosis. However, as a consequence of ultrasound attenuation, these parameters have increased to $89.7,100,100$, and $92.3 \%$, respectively. ${ }^{21}$

Other studies have investigated the use of computed tomography and magnetic resonance imaging to investigate hepatic steatosis. ${ }^{22}$ Nevertheless, both computed tomography and magnetic resonance imaging are expensive tests and require sedation of the child, which prevents its use in the routine investigation of NAFLD in the pediatric population. 22

Hepatic steatosis associated with increased serum level of alanine aminotransferase is a marker used for the

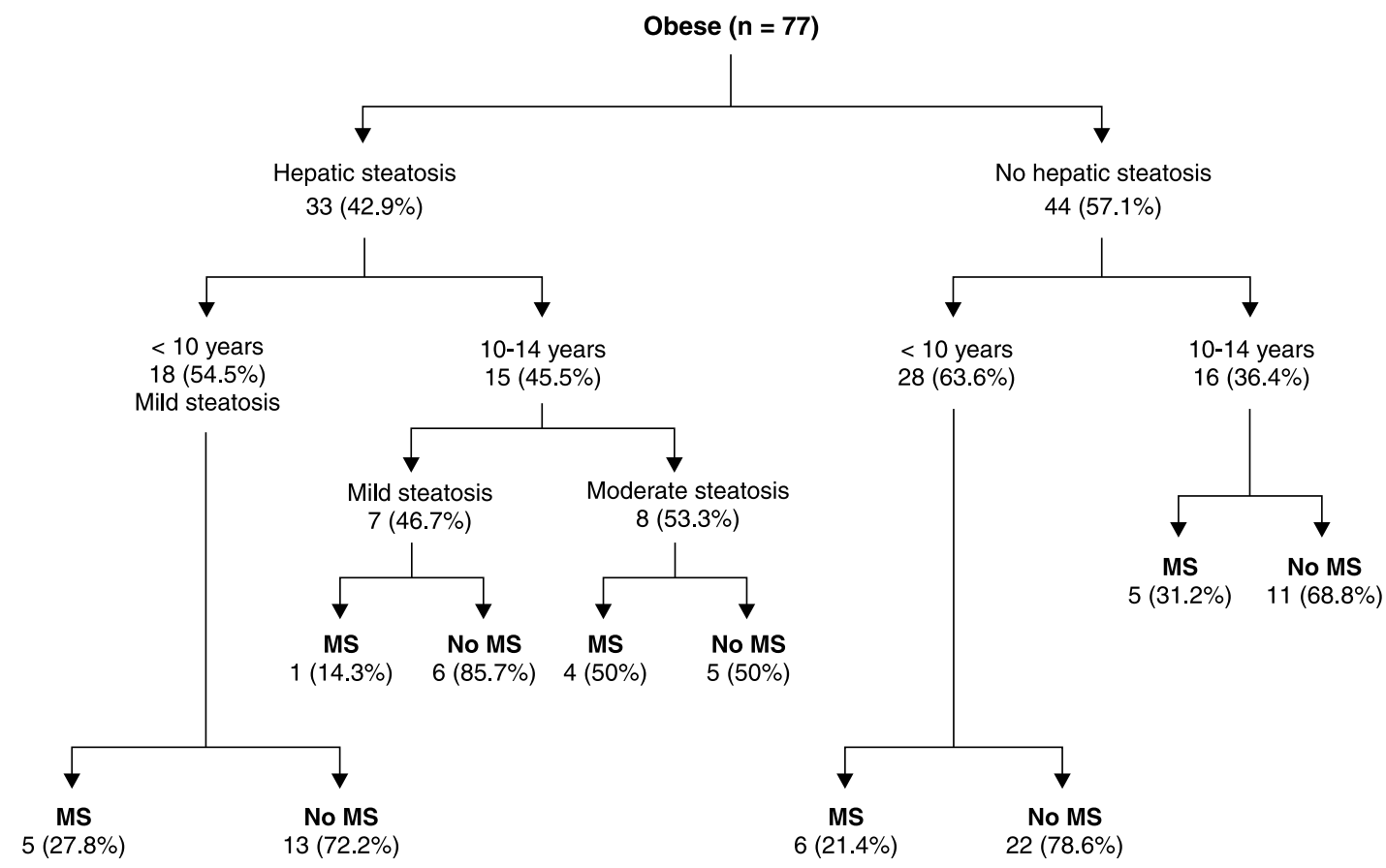

MS = metabolic syndrome.

Figure 1 - Distribution of hepatic steatosis and metabolic syndrome in 77 obese patients by age group 
presumptive diagnosis of steatohepatitis in the absence of histopathological investigation.6,23 In our study, $9.1 \%$ of children with steatosis diagnosed by liver ultrasound showed increase of alanine aminotransferase, a percentage lower than the one reported in the literature. 20

Franzese et al. ${ }^{20}$ reported that in children the serum levels of aminotransferase correlate with moderate or severe degrees of hepatic steatosis. Thus, it has been recommended that, even in the absence of alteration in aminotransferase serum levels, liver ultrasound is included in the overall evaluation of obese patients with the purpose of identifying mild cases of liver fat infiltration.

The predominance of males at a ratio of 2.7:1 in the present study was in agreement with data from other studies. ${ }^{6,11,22}$

The presence of acanthosis nigricans associated with hepatic steatosis was higher than that reported in the literature. ${ }^{19,24}$ Schwimmer et al. ${ }^{19}$ found acanthosis nigricans in $49 \%$ of 43 children undergoing liver biopsy for the diagnosis of NAFLD and considered that pediatricians should value this finding, which may evidence hyperinsulinemia.

It has been suggested that NAFLD is a component of the insulin resistance syndrome. ${ }^{25,26}$ In order to test this relationship, Fishbein et al. ${ }^{22}$ used magnetic resonance imaging to assess 39 obese patients aged 6 to 18 years old, with hepatomegaly and hypertransaminasemia. The authors identified a correlation of hepatic fat fraction with visceral adiposity, but the same was not true with regard to subcutaneous adiposity and degree of obesity. These findings suggest that, in addition to obesity, visceral adiposity plays a role in the pathogenesis of hepatic steatosis in children and, consequently, the insulin resistance syndrome seems to be present; however, further studies are needed to determine the relationship between hepatic steatosis, visceral adiposity, and other components of the metabolic syndrome.

Waist circumference correlates more with visceral adiposity than BMI and it has been considered a predictor of the insulin resistance syndrome, alteration in the serum levels of lipids and blood pressure in youths. ${ }^{25,26}$ In our study, we found a higher mean waist circumference among patients with hepatic steatosis. These data warrant and support the investigation of metabolic syndrome and hepatic steatosis in these children and adolescents.

As the determination of insulin resistance is not considered practical for clinical use, the International Diabetes Federation, 14 when defining the criteria for a definitive diagnosis of metabolic syndrome, considered waist circumference as a basic parameter that could be measured in a simple and practical manner, regardless of age and different parameters of the lipid profile.

Lee et al. ${ }^{25}$ compared 70 healthy African-American children with 97 Caucasian children and found that waist circumference correlated significantly with blood pressure measured during the day and at night, as well as with markers of dyslipidemia, regardless of race, even after adjustment of waist circumference for height.

The identification of increased blood pressure and alterations in the lipid profile, evidenced by decreased levels of HDL cholesterol in our series, enables us to raise the hypothesis of a relationship between hepatic steatosis and metabolic syndrome in this group according to the criteria suggested by Cook et al. ${ }^{13}$ The frequency of metabolic syndrome $(26.7 \%)$, regardless of the presence of hepatic steatosis, was lower than the rate of $42.4 \%$ reported by Buff et al. 27 in a study involving 59 children whose mean age was $10.9 \pm 0.48$ years in São Paulo, state of São Paulo, Brazil, using the same criteria.

\section{Conclusions}

NAFLD was a common condition in our sample, but it was not associated with increased serum levels of aminotransferase, a fact that draws attention to the need to use liver ultrasound in the routine investigation of obese children and adolescents.

The identification of a high frequency of metabolic syndrome among children and adolescents suggests that waist circumference measurement is important and should be considered when assessing Brazilian children because it is one of the diagnostic criteria of this syndrome, being associated with the presence of hepatic steatosis.

\section{Acknowledgements}

The authors would like to thank the physicians of Hospital Barão de Lucena, Recife, Brazil, Dr. Fernando José do Amaral, Head of the Radiology Center, Dr. Paula Salazar, Resident physician in Radiology, and Dr. Aline Borges Maciel, Resident Physician in Pediatrics for their priceless contribution to the present study.

\section{References}

1. MacPhee M. Global childhood obesity: how to curb an epidemic. J Pediatr Nurs. 2008;23:1-4.

2. Festi D, Colecchia A, Sacco T, Bondi M, Roda E, Marchesini G. Hepatic steatosis in obese patients: clinical aspects and prognostic significance. Obes Rev. 2004;5:27-42.

3. Roberts EA. Pediatric nonalcoholic fatty liver disease (NAFLD): a "growing" problem? J Hepatol. 2007;46:1133-42.

4. Lira AR, Oliveira FL, Escrivão MA, Colugnati FA, Taddei JA. Hepatic steatosis in a school population of overweight and obese adolescents. J Pediatria (Rio J). 2010;86:45-52.

5. Shoelson SE, Herrero L, Naaz A. Obesity, inflammation, and insulin resistance. Gatroenterology. 2007;132:2169-80

6. Papandreou D, Rousso I, Mavromichalis I. Update on non-alcoholic fatty liver disease in children. Clin Nutr. 2007;26:409-15. 
7. Pelekar NA, Naus R, Larson SP, Ward J, Harrison SA. Clinical model for distinguishing nonalcoholic steatohepatitis from simple steatosis in patients with nonalcoholic fatty liver disease. Liver Int. $2006 ; 26: 151-6$.

8. Nanda K. Non-alcoholic steatohepatitis in children. Pediatr Transplant. 2004;8:613-8

9. Moran JR, Grishan FK, Halter SA, Greene HL. Steatohepatitis in obese children: a cause of chronic liver dysfunction. Am J Gastroenterol. 1983;78:374-7.

10. Tominaga K, Kurata JH, Chen YK, Fujimoto E, Miyagawa S, Abe I, et al. Prevalence of fatty liver in Japanese children and relationship to obesity. An epidemiological ultrasonographic survey. Dig Dis Sci. 1995;40:2002-9.

11. Chan DF, Li AM, Chu WC, Chan MH, Wong EM, Liu EK, et al. Hepatic steatosis in obese Chinese children. Int J Obes Relat Metab Disord. 2004;28:1257-63.

12. Kuczmarski RJ, Ogden CL, Guo SS, Grummer-Strawn LM, Flegal KM, Mei Z, et al. 2000 CDC growth charts for the United States: Methods and development. Vital Health Stat 11. 2002:1-190.

13. Cook S, Weitzman M, Auinger P, Nguyen M, Dietz WH. Prevalence of a metabolic syndrome phenotype in adolescents: findings from the third National Health and Nutrition Examination Survey, 19881994. Arch Pediatr Adolesc Med. 2003;157:821-7.

14. International Diabetes Federation (IDF). The IDF consensus definition of the metabolic syndrome in children and adolescents. Brussels: IDF; 2007.

15. Sociedade Brasileira de Hipertensão (SBH). V Diretrizes Brasileiras de Hipertensão Arterial. Arq Bras Cardiol. 2007;89:e24-79.

16. Maitra SK, Rowland Payne CM. The obesity syndrome and acanthosis nigricans. Acanthosis nigricans is a common cosmetic problem providing epidemiological clues to the obesity syndrome, the insulin-resistance syndrome, the thrifty metabolism, dyslipdaemia hypertension and diabetes mellitus type II. J Cosmet Dermatol. 2004;3:202-10.

17. Kwiterovich PO Jr. Biochemical, clinical, epidemiologic, genetic, and pathologic data in the pediatric age group relevant to the cholesterol hypothesis. Pediatrics. 1986;78:349-62.

18. Seist G, Schiele F, Galteau M, Panek E, Steinmetz J, Fagnani F, et al. Aspartate aminotransferase and alanine aminotransferase activities in plasma: statistical distributions, individual variations, and reference values. Clin Chem. 1975;21:1077-87.
19. Schwimmer JB, Deutsch R, Rauch JB, Behling R, Newbury R, Lavine JE. Obesity, insulin resistance, and other clinicopathological correlates of pediatric nonalcoholic fatty liver disease. J Pediatr. 2003;143:500-5

20. Franzese A, Vajro P, Argenziano A, Puzziello A, Iannucci MP, Saviano $M C$, et al. Liver involvement in obese children. Ultrasonography and liver enzyme levels at diagnosis and during follow-up in an Italian population. Dig Dis Sci. 1997;42:1428-32.

21. Palmentieri B, de Sio I, La Mura V, Masarone M, Vecchione $R$, Bruno $S$, et al. The role of bright liver echo pattern on ultrasound B-mode examination in the diagnosis of liver steatosis. Dig Liver Dis. $2006 ; 38: 485-9$

22. Fishbein $\mathbf{M H}$, Mogren $\mathrm{C}$, Gleason $\mathrm{T}$, Stevens WR. Relationship of hepatic steatosis to adipose tissue distribution in pediatric nonalcoholic fatty liver disease. J Pediatr Gastroenterol Nutr. 2006;42:83-8.

23. Patton HM, Sirlin C, Behling C, Middleton M, Schwimmer JB, Lavine JE. Pediatric nonalcoholic fatty liver disease: a critical appraisal of current data and implications for future research. J Pediatr Gastroenterol Nutr. 2006;43:413-27.

24. Rashid M, Roberts EA. Nonalcoholic steatohepatitis in children. J Pediatr Gastroenterol Nutr. 2000;30:48-53.

25. Lee S, Bacha F, Arslanian SA. Waist circumference, blood pressure, and lipid components of the metabolic syndrome. J Pediatr. 2006;149:809-16.

26. Weiss R, Kaufman FR. Metabolic complications of childhood obesity: identifying and mitigating the risk. Diabetes Care. 2008;31 Suppl 2:S310-6.

27. Buff CG, Ramos E, Souza FI, Sarni RO. Freqüência de síndrome metabólica em crianças e adolescentes com sobrepeso e obesidade. Rev Paul Pediatr. 2007;25:221-6.

Correspondence:

Maria Amélia Soares de Melo Duarte

Rua Rosângela Cunha C. Wanderley, 164/601 - Piedade

CEP 54420-180 - Jaboatão dos Guararapes, PE - Brazil

Tel.: + 55 (81) 3468.5684

E-mail: ameliaduarte@hotmail.com 